\section{Hypothermia after Reperfusion Suppresses Aggravation of Acute Brain Edema following Transient Forebrain Ischemia in Mice}

\author{
Masaru Doshi,* Etsuka Higuchi, and \\ Yutaka Hirashima \\ Department of Physiological Chemistry, Faculty of Pharma- \\ ceutical Sciences, Teikyo University, 1091-1 Suarashi, Midori- \\ ku, Sagamihara 252-5195, Japan
}

(Received September 8, 2010; Accepted October 21, 2010)

In our previous paper, we reported that spontaneous hypothermia (HT) during ischemia protects against delayed neuronal death in the hippocampus but not against acute brain edema following transient forebrain ischemia, which is induced by occlusion of the bilateral common carotid arteries (BCCA) in $\mathrm{C57BL} / 6 \mathrm{~J}$ mice. We here demonstrate that artificial HT after reperfusion (rHT) suppresses the aggravation of acute brain edema in the BCCA occlusion C57BL/6J mouse model. Our results suggest that mechanisms regulated by $\mathrm{rHT}$ are involved in the attenuation of acute brain edema after reperfusion in this model of cerebral ischemia.

Key words — cerebral ischemia, brain edema, hypothermia, reperfusion, mouse

\section{INTRODUCTION}

Transient forebrain ischemia, which is induced by occlusion of the bilateral common carotid arteries (BCCA) in mice, causes delayed neuronal death in the hippocampus and is widely known as a model of brain injury following transient cardiac arrest. ${ }^{1)}$ The BCCA occlusion C57BL/6J mouse model is a valuable tool for investigating the molecular mechanism of delayed neuronal death following cerebral ischemia. ${ }^{2-4)}$ Recently, we have demonstrated that acute brain edema, one of the most important disor-

\footnotetext{
*To whom correspondence should be addressed: Department of Physiological Chemistry, Faculty of Pharmaceutical Sciences, Teikyo University, 1091-1 Suarashi, Midori-ku, Sagamihara 252-5195, Japan. Tel.: \& Fax: +81-42-685-3745; E-mail: mdoshi@pharm.teikyo-u.ac.jp
}

— Research Letter -

ders following cerebral ischemia, occurs in the forebrain of the C57BL/6J mouse model, which may be useful for studying the molecular mechanism and developing novel therapeutic agents for ischemic brain edema. ${ }^{5)}$

Hypothermia (HT) is one of the most putative neuroprotective therapies for brain injury caused by cardiac arrest in humans, ${ }^{6}$ ) although there is as yet no evidence of such an effect of HT on cerebrovascular diseases from a large-scale clinical trial. Several studies have shown that HT, which was monitored by measuring rectal temperature, protects against delayed neuronal death and infarction, ${ }^{7,8)}$ but few studies have investigated the effects of HT on brain edema in mouse models of cerebral ischemia. We have recently found that spontaneous HT during ischemia protects against delayed neuronal death but not against acute brain edema in the BCCA occlusion C57BL/6J mouse model. ${ }^{5)}$ However, we speculated that the ineffectiveness of HT during ischemia against acute brain edema is due to the immediate recovery of rectal temperature after reperfusion at room temperature in the BCCA occlusion C57BL/6J mouse model. Therefore, we investigated the effect of artificial HT after reperfusion (rHT) on acute brain edema in the BCCA occlusion $\mathrm{C} 57 \mathrm{BL} / 6 \mathrm{~J}$ mouse model.

\section{MATERIALS AND METHODS}

Animals — All the procedures were carried out in strict accordance with the guidelines of the Committee of Animal Care and Experiments of Teikyo University. Male C57BL/6J mice were purchased from Charles River Japan (Yokohama, Japan). Eight- to ten-week-old mice weighing between 25 and $30 \mathrm{~g}$ were used in this study. The mice were housed in plastic cages in the animal laboratory of Teikyo University and fed a chow diet (CRF-1, Oriental Yeast Co., Tokyo, Japan).

Induction of Transient Forebrain Ischemia Forebrain ischemia was induced by BCCA occlusion with clips (Mizuho Co., Ltd., Tokyo, Japan) for 15 min under $1 \%$ halothane (Takeda Pharmaceutical Co., Ltd., Osaka, Japan) anesthesia in air using a face mask. Rectal temperature was monitored using a digital thermometer (TC-1, BrainScience Idea Co., Ltd., Osaka, Japan) and maintained at $37 \pm 0.5^{\circ} \mathrm{C}$ with a heating blanket (normothermia, 
NT). The control mice underwent a sham operation without BCCA occlusion under halothane anesthesia for $15 \mathrm{~min}$.

Induction of Hypothermia — The mice were divided into two groups in terms of the control of rectal temperature after reperfusion. HT during ischemia was spontaneously induced by removing the heating blanket, ${ }^{5)}$ and the mice were allowed to recover from anesthesia at room temperature (23$25^{\circ} \mathrm{C}$ ) until $1 \mathrm{hr}$ after reperfusion. In contrast, rHT was induced by placing the mice on a refrigerant, which was maintained at $18-19^{\circ} \mathrm{C}$ until $1 \mathrm{hr}$ after reperfusion in a styrofoam box.

Measurement of Water Content in Forebrain — The water content in the forebrain was determined by the weight differences between the wet and dry samples, as described previously. ${ }^{5)}$

Statistical Analysis — Analysis of variance (ANOVA) of repeated measures was used for the comparison of the changes in rectal temperature between the HT and rHT groups. Two-way ANOVA was used to analyze the effect of rectal temperature after reperfusion on the water content in the forebrain in experiments on ischemia $15 \mathrm{~min}$ and $1 \mathrm{hr}$ after reperfusion (I-R), and an unpaired Student's $t$-test for comparison between the control and I-R groups, or the HT and rHT groups. A $p$ value of less than 0.05 was considered statistically significant in all tests.

\section{RESULTS}

In our previous study, both NT and HT mice recovered from anesthesia at room temperature after reperfusion in the BCCA occlusion C57BL/6J mouse model. ${ }^{5)}$ Consequently, we first investigated the changes in the rectal temperature of NT and HT mice until $24 \mathrm{hr}$ after BCCA occlusion and reperfusion. As shown in Fig. 1, the rectal temperature of the HT mice $1 \mathrm{hr}$ after reperfusion was already the same as that of the NT mice. The changes in the rectal temperature of the control mice were similar to those of the I-R mice (data not shown). This result indicates that the rectal temperature of the NT mice decreases after reperfusion and that of the HT mice recovers from HT during ischemia to NT mice levels within $1 \mathrm{hr}$ after reperfusion.

The water content in the forebrain $1 \mathrm{hr}$ after reperfusion reached a peak in the BCCA occlusion C57BL/6J mouse model. ${ }^{5)}$ This finding is the same as that in the HT mice (data not shown). Con-

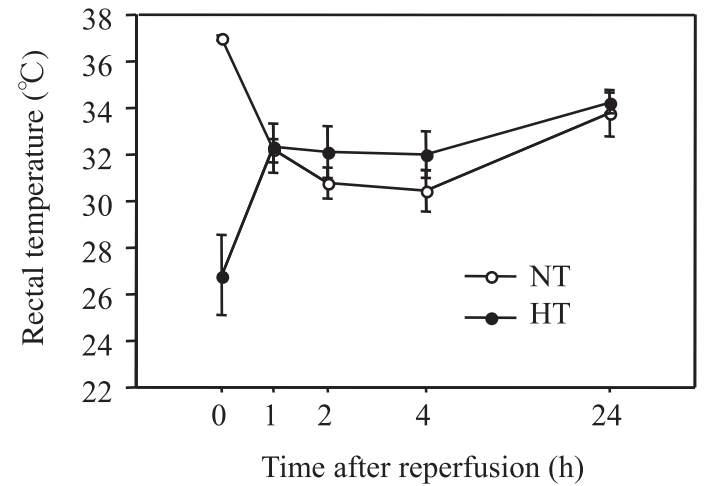

Fig. 1. Changes in Rectal Temperature after Reperfusion in the BCCA Occlusion C57BL/6J Mouse Model

Forebrain ischemia was induced by BCCA occlusion for $15 \mathrm{~min}$ under $1 \%$ halothane anesthesia. NT and HT were induced as described in the Materials and Methods. The rectal temperature at $0,1,2$, 4 , or $24 \mathrm{hr}$ after reperfusion was measured using a digital thermometer. Data are expressed as mean \pm S.E. $(n=6)$.

sequently, to evaluate the effect of rHT on acute brain edema after reperfusion in the BCCA occlusion C57BL/6J mouse model, we next compared brain water content $1 \mathrm{hr}$ after reperfusion between the HT and rHT mice. The changes in rectal temperature after reperfusion in the HT and rHT mice are shown in Fig. 2A. The patterns of the change in rectal temperature were significantly different between the HT and rHT mice (control: $F(2,8)=5.824$, $p=0.0275$, I-R: $F(2,24)=16.681, p<0.0001)$, but no differences were observed between the control and I-R mice in both groups (HT: $F(2,16)=0.848$, $p=0.4468$, rHT: $F(2,16)=1.572, p=0.238)$. Under these conditions for monitoring rectal temperature, we then compared the water content $1 \mathrm{hr}$ after reperfusion (Fig. 2B). The water content $1 \mathrm{hr}$ after reperfusion was significantly higher in both the HT and rHT mice than in the control mice $(p<0.05)$. However, the water content $1 \mathrm{hr}$ after reperfusion was significantly lower in the rHT mice than in the HT mice. We also confirmed that water content $2 \mathrm{hr}$ after reperfusion in rHT mice was equal to that in the control mice (rHT: $78.71 \pm 0.12 \%$, control: $78.77 \pm 0.07 \%$ ), indicating that the progression of brain edema formation in rHT mice was not delayed by rHT. Furthermore, we have shown an increase in brain water content during BCCA occlusion in a previous study. ${ }^{5)}$ These results indicate that rHT suppresses the additional increase in brain water content after reperfusion, but not the increase in brain water content during BCCA occlusion. 
A

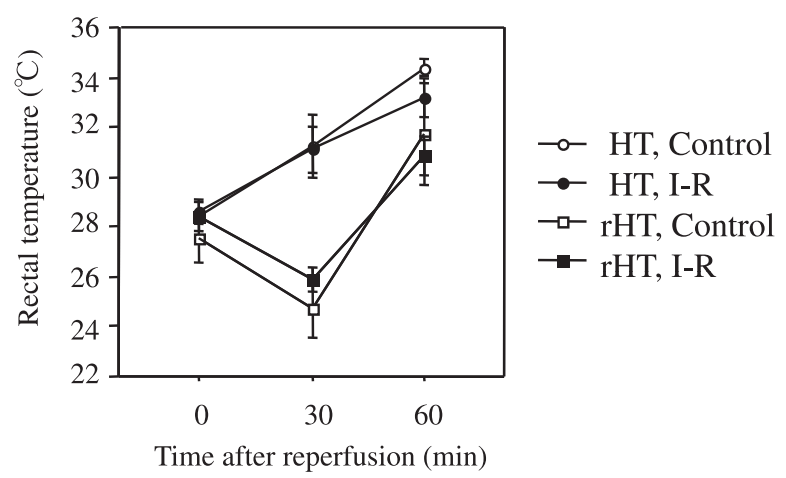

B

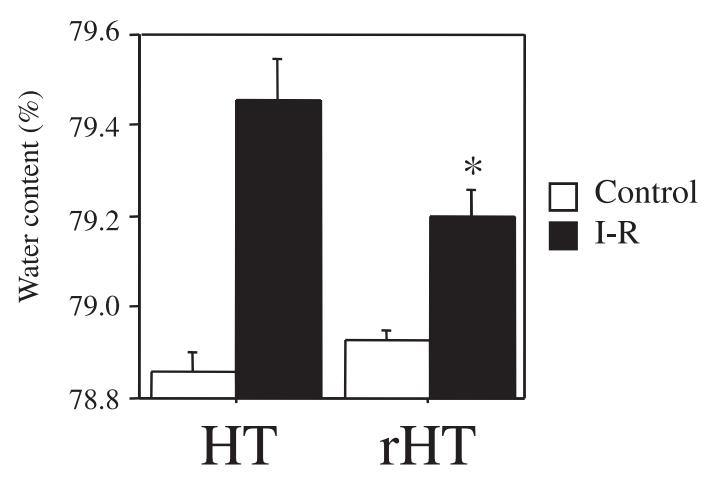

Fig. 2. Effect of HT after Reperfusion on Occurrence of Brain Edema in the BCCA Occlusion C57BL/6J Mouse Model

Forebrain ischemia was induced by BCCA occlusion for $15 \mathrm{~min}$ under $1 \%$ halothane anesthesia in both $\mathrm{HT}$ and $\mathrm{rHT}$ mice, as described in the Materials and Methods. The control mice (Control) underwent a sham operation without BCCA occlusion under halothane anesthesia for $15 \mathrm{~min}$. The rectal temperature was monitored 0,30 , and $60 \mathrm{~min}$ after reperfusion (A). The water content in the forebrain treated with BCCA occlusion for $15 \mathrm{~min}$ and I-R was measured (B). Data are expressed as mean \pm S.E. (control: $n=3$, I-R: $n=7$ ). Statistical analysis was performed by two-way ANOVA and the unpaired Student's $t$-test $\left({ }^{*} p<0.05 v s . \mathrm{HT}\right)$

\section{DISCUSSION}

NT during ischemia is essential for the induction of delayed neuronal death in the hippocampus of the BCCA occlusion C57BL/6J mouse model. ${ }^{9}{ }^{9}$ In addition, because rectal temperature decreases spontaneously under halothane anesthesia without regard to the induction of ischemia in mice, ${ }^{5)}$ controlled NT during anesthesia is quite normal in experiments on cerebral ischemia. ${ }^{10,11)}$ We found that rectal temperature shows no difference between NT and HT mice in the duration of occurrence of acute brain edema (Fig. 1). The immediate recovery of rectal temperature after reperfusion in HT mice was consistent with our speculation on the ineffective- ness of HT during ischemia to treat acute brain edema. Therefore, it is necessary to precisely monitor and distinguish rectal temperatures during ischemia and after reperfusion to investigate the effects of HT on acute brain edema in the BCCA occlusion C57BL/6J mouse model.

In this study, we have clearly shown that rHT suppresses the aggravation of acute brain edema following transient forebrain ischemia in C57BL/6J mice (Fig. 2B). On the other hand, we previously demonstrated that HT only during ischemia has no effect on acute brain edema in the same model of cerebral ischemia. ${ }^{5)}$ These findings suggest that the aggravation of brain edema after reperfusion is caused by mechanisms of brain edema formation that are different from those of the occurrence of brain edema during BCCA occlusion. Although reactive oxygen species and many inflammatory mediators such as cytokines and arachidonate metabolites are believed to perhaps be involved in the pathogenesis of ischemic brain injuries, ${ }^{12,13}$ the mechanisms of brain edema formation remain unclear. Therefore, elucidation of the mechanisms regulated by $\mathrm{rHT}$ in the $\mathrm{C} 57 \mathrm{BL} / 6 \mathrm{~J}$ mouse model is valuable for understanding the pathogenesis of acute brain edema following transient cerebral ischemia.

HT during ischemia protects against delayed neuronal death in the hippocampus. ${ }^{2,5)}$ Here, we demonstrated that rHT prevents the occurrence of acute brain edema following transient forebrain ischemia. Therefore, these findings indicate that the duration of HT differs depending on the pathology following cerebral ischemia. Further experimental studies should be performed to establish a standard method for the clinical application of therapeutic HT because there are still several controversial issues in the development of cooling techniques and in the determination of optimal duration and temperature. ${ }^{14)}$

Acknowledgement This work was partially supported by a Grant-in-Aid for Scientific Research from the Ministry of Education, Culture, Sports, Science and Technology of Japan (20591696).

\section{REFERENCES}

1) Kawase, M., Murakami, K., Fujimura, M., MoritaFujimura, Y., Gasche, Y., Kondo, T., Scott, R. W. and Chan, P. H. (1999) Exacerbation of delayed cell 
injury after transient global ischemia in mutant mice with $\mathrm{CuZn}$ superoxide dismutase deficiency. Stroke, 30, 1962-1968.

2) Yang, G., Kitagawa, K., Matsushita, K., Mabuchi, T., Yagita, Y., Yanagihara, T. and Matsumoto, M. (1997) C57BL/6 strain is most susceptible to cerebral ischemia following bilateral common carotid occlusion among seven mouse strains: selective neuronal death in the murine transient forebrain ischemia. Brain Res., 752, 209-218.

3) Tajiri, S., Oyadomari, S., Yano, S., Morioka, M., Gotoh, T., Hamada, J.-I., Ushio, Y. and Mori, M. (2004) Ischemia-induced neuronal cell death is mediated by the endoplasmic reticulum stress pathway involving CHOP. Cell Death Differ., 11, 403-415.

4) Wu, C., Zhan, R.-Z., Qi, S., Fujihara, H., Taga, K. and Shimoji, K. (2001) A forebrain ischemic preconditioning model established in C57Black/Crj6 mice. J. Neurosci. Methods, 107, 101-106.

5) Doshi, M., Kuwatori, Y., Ishii, Y., Sasahara, M. and Hirashima, Y. (2009) Hypothermia during ischemia protects against neuronal death but not acute brain edema following transient forebrain ischemia in mice. Biol. Pharm. Bull., 32, 1957-1961.

6) Benard, S. A., Gray, T. W., Buist, M. D., Jones, B. M., Silvester, W., Gutteridge, G. and Smith, K. (2002) Treatment of comatose survivors of out-ofhospital cardiac arrest with induced hypothermia. $N$. Engl. J. Med., 346, 557-563.

7) Maier, C. M., Ahern, K., Cheng, M. L., Lee, J. E., Yenari, M. A. and Steinberg, G. K. (1998) Optimal depth and duration of mild hypothermia in a focal model of transient cerebral ischemia. Stroke, 29, 2171-2180.
8) Tsuchiya, D., Hong, S., Suh, S. W., Kayama, T., Panter, S. S. and Weinstein, P. R. (2002) Mild hypothermia reduces zinc translocation, neuronal cell death, and mortality after transient global ischemia in mice. J. Cereb. Blood Flow Metab., 22, 12311238.

9) Ohtaki, H., Nakamachi, T., Dohi, K., Yofu, S., Hodoyama, K., Matsunaga, M., Aruga, T. and Shioda, S. (2006) Controlled normothermia during ischemia is important for the induction of neuronal cell death after global ischemia in mouse. Acta Neurochir. (Wien), 96, 249-253.

10) Marcheselli, V. L., Hong, S., Lukiw, W. J., Tian, H. T., Gronert, K., Musto, A., Hardy, M., Gimenez, J. M., Chiang, N., Serhan, C. N. and Bazan, N. G. (2003) Novel docosanoids inhibit brain ischemiareperfusion-mediated leukocyte infiltration and proinflammatory gene expression. J. Biol. Chem., 278, 43807-43817.

11) Umemura, K., Kato, I., Hirashima, Y., Ishii, Y., Inoue, T., Aoki, J., Kono, N., Oya, T., Hayashi, N., Hamada, H., Endo, S., Oda, M., Arai, H., Kinouchi, H. and Hiraga, K. (2007) Neuroprotective role of transgenic PAF-acetylhydrolase II in mouse models of focal cerebral ischemia. Stroke, 38, 1063-1068.

12) Barone, F. C. and Feuerstein, G. Z. (1999) Inflammatory mediators and stroke: new opportunities for novel therapeutics. J. Cereb. Blood Flow Metab., 19, 819-834.

13) Chan, P. H. (1996) Role of oxidants in ischemic brain damage. Stroke, 27, 1124-1129.

14) Liu, L., Yenari, M. A. and Ding, Y. (2009) Clinical application of therapeutic hypothermia in stroke. Neurol. Res., 31, 331-335. 